\title{
On Intra-Regular Left Almost Semihypergroups with Pure Left Identity
}

\author{
Naveed Yaqoob, ${ }^{1}$ Piergiulio Corsini, ${ }^{2}$ and Faisal Yousafzai ${ }^{3}$ \\ ${ }^{1}$ Department of Mathematics, Quaid-i-Azam University, Islamabad, Pakistan \\ ${ }^{2}$ Department of Civil Engineering and Architecture, Via delle Scienze 206, Udine, Italy \\ ${ }^{3}$ Department of Mathematics, COMSATS Institute of Information Technology, Attock, Pakistan
}

Correspondence should be addressed to Naveed Yaqoob; nayaqoob@ymail.com

Received 24 January 2013; Accepted 4 April 2013

Academic Editor: Feng Feng

Copyright (C) 2013 Naveed Yaqoob et al. This is an open access article distributed under the Creative Commons Attribution License, which permits unrestricted use, distribution, and reproduction in any medium, provided the original work is properly cited.

We characterize intra-regular LA-semihypergroups by using the properties of their left and right hyperideals, and we investigate some useful conditions for an LA-semihypergroup to become an intra-regular LA-semihypergroup.

\section{Introduction}

Kazim and Naseeruddin [1] introduced the concept of left almost semigroups (abbreviated as LA-semigroups) and right almost semigroups (abbreviated as RA-semigroups). They generalized some useful results of semigroup theory. Later, Mushtaq [2,3] and others further investigated the structure and added many useful results to the theory of LAsemigroups; see also [4-9]. An LA-semigroup is the midway structure between a commutative semigroup and a groupoid. It nevertheless possesses many interesting properties which we usually find in commutative and associative algebraic structures. Mushtaq and Yusuf produced useful results [10] on locally associative LA-semigroups in 1979. In this structure, they defined powers of an element and congruences using these powers. There are several results which have been added to the theory of LA-semigroups by Mushtaq, Shabir, Aslam, Davvaz, Madad, Hila, Chinram, Holgate, Jezek, Protic, and many other researchers.

Hyperstructure theory was introduced in 1934, when Marty [11] defined hypergroups, began to analyze their properties, and applied them to groups. In the following decades and nowadays, a number of different hyperstructures are widely studied from the theoretical point of view. Nowadays, hyperstructures have a lot of applications to several domains of mathematics and computer science, and they are studied in many countries of the world. In a classical algebraic structure, the composition of two elements is an element, while in an algebraic hyperstructure, the composition of two elements is a set. A lot of papers and several books have been written on hyperstructure theory; see [12,13]. A recent book on hyperstructures [14] points out on their applications in rough set theory, cryptography, codes, automata, probability, geometry, lattices, binary relations, graphs, and hypergraphs. Another book [15] is devoted especially to the study of hyperring theory. The volume ends with an outline of applications in chemistry and physics, analyzing several special kinds of hyperstructures: $e$-hyperstructures and transposition hypergroups. Many authors studied different aspects of semihypergroups, for instance, Aslam et al. [16-20], Corsini et al. [21, 22], Davvaz et al. [23, 24], Hasankhani [25], Hila et al. [26], Leoreanu [27], Onipchuk [28], and Yaqoob et al. [2934].

Recently, Hila and Dine [35] introduced the notion of LAsemihypergroups as a generalization of semigroups, semihypergroups, and LA-semigroups. They investigated several properties of hyperideals of LA-semihypergroup and defined the topological space and studied the topological structure of LA-semihypergroups using hyperideal theory.

In this paper, we will prove some results on intra-regular LA-semihypergroups. 


\section{Basic Definitions and Examples}

In this section, we recall certain definitions and results needed for our purpose.

Definition 1. A map $\circ: H \times H \rightarrow \mathscr{P}^{*}(H)$ is called hyperoperation or join operation on the set $H$, where $H$ is a nonempty set and $\mathscr{P}^{*}(H)=\mathscr{P}(H) \backslash\{\emptyset\}$ denotes the set of all nonempty subsets of $H$. A hypergroupoid is a set $H$ together with a (binary) hyperoperation.

If $A$ and $B$ are two nonempty subsets of $H$, then we denote

$$
\begin{gathered}
A \circ B=\bigcup_{a \in A, b \in B} a \circ b, \\
a \circ A=\{a\} \circ A, \\
a \circ B=\{a\} \circ B .
\end{gathered}
$$

Definition 2 (see [35]). A hypergroupoid $(H, \circ)$ is called an LA-semihypergroup if for all $x, y, z \in H$,

$$
(x \circ y) \circ z=(z \circ y) \circ x .
$$

The law $(x \circ y) \circ z=(z \circ y) \circ x$ is called a left invertive law.

Example 3. Let $H=\{x, y, z, w, t\}$ with the binary hyperoperation defined below:

\begin{tabular}{c|ccccc}
$\circ$ & $x$ & $y$ & $z$ & $w$ & $t$ \\
\hline$x$ & $x$ & $x$ & $x$ & $x$ & $x$ \\
$y$ & $x$ & $\{z, t\}$ & $z$ & $\{x, w\}$ & $\{z, t\}$ \\
$z$ & $x$ & $z$ & $z$ & $\{x, w\}$ & $z$ \\
$w$ & $x$ & $\{x, w\}$ & $\{x, w\}$ & $w$ & $\{x, w\}$ \\
$t$ & $x$ & $\{y, t\}$ & $z$ & $\{x, w\}$ & $\{y, t\}$
\end{tabular}

Clearly, $H$ is not a semihypergroup because $\{y, z, t\}=(t \circ$ $t) \circ y \neq t \circ(t \circ y)=\{y, t\}$. Thus, $H$ is an LA-semihypergroup because the elements of $H$ satisfy the left invertive law.

Example 4. Let $H=\mathbb{Z}$. If we define $x \circ y=y-x+3 \mathbb{Z}$, where $x, y \in \mathbb{Z}$. Then, $(H, \circ)$ becomes an LA-semihypergroup as

$$
\begin{aligned}
(x \circ y) \circ z & =z-(x \circ y)+3 \mathbb{Z} \\
& =z-(y-x+3 \mathbb{Z})+3 \mathbb{Z} \\
& =z-y+x-3 \mathbb{Z}+3 \mathbb{Z} \\
& =x-y+z-3 \mathbb{Z}+3 \mathbb{Z} \\
& =x-(y-z+3 \mathbb{Z})+3 \mathbb{Z} \\
& =x-(z \circ y)+3 \mathbb{Z} \\
& =(z \circ y) \circ x .
\end{aligned}
$$

This implies that $(x \circ y) \circ z=(z \circ y) \circ x$ holds for all $x, y, z \in$ $H=\mathbb{Z}$, and also it is clear that $(x \circ y) \circ z \neq x \circ(y \circ z)$. Hence $(H, \circ)$ is an LA-semihypergroup.

Every LA-semihypergroup satisfies the law

$$
(x \circ y) \circ(z \circ w)=(x \circ z) \circ(y \circ w)
$$

for all $x, y, z, w \in H$. This law is known as medial law (cf. [35]).

Definition 5. Let $H$ be an LA-semihypergroup. An element $e \in H$ is called

(i) left identity (resp., pure left identity) if for all $a \in H$, $a \in e \circ a$ (resp., $a=e \circ a)$,

(ii) right identity (resp., pure right identity) if for all $a \in$ $H, a \in a \circ e$ (resp., $a=a \circ e)$,

(iii) identity (resp., pure identity) if for all $a \in H, a \in e$ 。 $a \cap a \circ e$ (resp., $a=e \circ a \cap a \circ e)$.

Example 6. Let $H=\{x, y, z, w\}$ with the binary hyperoperation defined below:

\begin{tabular}{c|cccc}
$\circ$ & $x$ & $y$ & $z$ & $w$ \\
\hline$x$ & $x$ & $y$ & $z$ & $w$ \\
$y$ & $z$ & $\{y, z\}$ & $\{y, z\}$ & $w$ \\
$z$ & $y$ & $\{y, z\}$ & $\{y, z\}$ & $w$ \\
$w$ & $w$ & $w$ & $w$ & $H$
\end{tabular}

Clearly $H$ is an LA-semihypergroup because the elements of $H$ satisfy the left invertive law. Here, $x$ is a pure left identity because for all $a \in H, a=x \circ a$. And, in Example 6, one can see that $t$ is a left identity but not a pure left identity.

Lemma 7. Let $H$ be an LA-semihypergroup with pure left identitye. Then, $x \circ(y \circ z)=y \circ(x \circ z)$ holds for all $x, y, z \in H$.

Proof. Let $H$ be an LA-semihypergroup with pure left identity $e$. Then, for all $x, y, z \in H$ and by medial law, we have

$$
\begin{aligned}
x \circ(y \circ z) & =(e \circ x) \circ(y \circ z) \\
& =(e \circ y) \circ(x \circ z)=y \circ(x \circ z) .
\end{aligned}
$$

This completes the proof.

Lemma 8. Let $H$ be an LA-semihypergroup with pure left identity e. Then, $(x \circ y) \circ(z \circ w)=(w \circ y) \circ(z \circ x)$ holds for all $x, y, z, w \in H$.

Proof. Let $H$ be an LA-semihypergroup with pure left identity $e$. Then, for all $x, y, z, w \in H$ and by medial law, we have

$$
\begin{aligned}
(x \circ y) \circ(z \circ w) & =((e \circ x) \circ y) \circ((e \circ z) \circ w) \\
& =((y \circ x) \circ e) \circ((w \circ z) \circ e) \\
& =((y \circ x) \circ(w \circ z)) \circ(e \circ e) \\
& =((e \circ e) \circ(w \circ z)) \circ(y \circ x) \\
& =(e \circ(w \circ z)) \circ(y \circ x) \\
& =(w \circ z) \circ(y \circ x)=(w \circ y) \circ(z \circ x) .
\end{aligned}
$$

This completes the proof.

The law $(x \circ y) \circ(z \circ w)=(w \circ y) \circ(z \circ x)$ is called a paramedial law. 
Definition 9. Let $H$ be an LA-semihypergroup. A nonempty subset $A$ of $H$ is called a sub LA-semihypergroup of $H$ if $x$ 。 $y \subseteq A$ for every $x, y \in A$.

Definition 10. A subset $I$ of an LA-semihypergroup $H$ is called a right (left) hyperideal of $H$ if $I \circ H \subseteq I(H \circ I \subseteq I)$ and is called a hyperideal if it is two-sided hyperideal.

Definition 11. By a bi-hyperideal of an LA-semihypergroup $H$, we mean a sub LA-semihypergroup $B$ of $H$ such that $(B \circ H) \circ B \subseteq B$.

Definition 12. A sub LA-semihypergroup $B$ of $H$ is called a $(1,2)$-hyperideal of $H$ if $(B \circ H) \circ B^{2} \subseteq B$.

It is easy to note that each right hyperideal is a bihyperideal. If $E\left(B_{H}\right)$ denotes the set of all idempotent subsets of $H$ with pure left identity $e$, then $E\left(B_{H}\right)$ forms a hypersemilattice structure. The intersection of any set of bihyperideals of an LA-semihypergroup $H$ is either empty or a bi-hyperideal of $H$.

Definition 13. A sub LA-semihypergroup $T$ of $H$ is called an interior hyperideal of $H$ if $(H \circ T) \circ H \subseteq T$.

Definition 14. A nonempty subset $Q$ of an LA-semihypergroup $H$ is called a quasi-hyperideal of $H$ if $Q \circ H \cap H \circ Q \subseteq Q$.

Lemma 15. If $H$ is an LA-semihypergroup with left identitye, then $H \circ H=H$.

Proof. If $H$ is an LA-semihypergroup with left identity $e$, then $x \in H$ implies that

$$
x \in e \circ x \subseteq H \circ H \text { and so } H \subseteq H \circ H .
$$

That is, $H=H \circ H$.

Corollary 16. If $H$ is an LA-semihypergroup with pure left identitye, then $\mathrm{H} \circ \mathrm{H}=\mathrm{H}$ and $\mathrm{H}=e \circ \mathrm{H}=\mathrm{H} \circ \mathrm{e}$.

Proof. The proof is similar to the proof of Lemma 15.

In an LA-semigroup, every right identity becomes a left identity. But in an LA-semihypergroup, every right identity needs not to be a left identity. For this, let $H=\{x, y, z\}$ with the binary hyperoperation defined below:

\begin{tabular}{c|ccc}
$\circ$ & $x$ & $y$ & $z$ \\
\hline$x$ & $\{x, z\}$ & $z$ & $\{y, z\}$ \\
$y$ & $\{y, z\}$ & $z$ & $z$ \\
$z$ & $\{y, z\}$ & $\{y, z\}$ & $\{y, z\}$
\end{tabular}

Clearly, $H$ is not a semihypergroup because $\{y, z\}=(y \circ$ $y) \circ z \neq y \circ(y \circ z)=\{z\}$. Thus, $H$ is an LA-semihypergroup because the elements of $H$ satisfy the left invertive law. Here, $x$ is a right identity but not a left identity. However, if an LAsemihypergroup $H$ has a pure right identity $e$, then $e$ becomes a pure left identity. For this, consider $b \in H$ and $e$ be a pure right identity of $H$ then;

$$
b=b \circ e=(b \circ e) \circ e=(e \circ e) \circ b=e \circ b .
$$

This shows that in an LA-semihypergroup, every pure right identity becomes a pure left identity. Every LA-semihypergroup with pure right identity becomes a commutative hypermonoid.

Theorem 17. An LA-semihypergroup $H$ is a semihypergroup if and only if $a \circ(b \circ c)=(c \circ b) \circ a$ holds for all $a, b, c \in H$.

Proof. Let $H$ be a semihypergroup. Then, we have $(a \circ b) \circ c=$ $a \circ(b \circ c)$ for all $a, b, c \in H$, but $(a \circ b) \circ c=(c \circ b) \circ a$; thus,

$$
a \circ(b \circ c)=(c \circ b) \circ a \quad \forall a, b, c \in H \text {. }
$$

On the other hand, suppose that $a \circ(b \circ c)=(c \circ b) \circ a$ holds for all $a, b, c \in H$. Since $H$ is an LA-semihypergroup, therefore

$$
a \circ(b \circ c)=(c \circ b) \circ a=(a \circ b) \circ c .
$$

Thus, $H$ is a semihypergroup. This completes the proof.

\section{Intra-Regular LA-Semihypergroups}

In this section, we will characterize intra-regular LA-semihypergroup by using the properties of left and right hyperideals.

Definition 18. Let $H$ be an LA-semihypergroup and $a \in H$. Then, $a$ is said to be regular if there exist an element $x \in H$ such that $a \in(a \circ x) \circ a$. The LA-semihypergroup $H$ is called regular if every element of $H$ is regular.

Definition 19. An element $a$ of an LA-semihypergroup $H$ is called an intra-regular element if there exist $x, y \in H$ such that $a \in\left(x \circ a^{2}\right) \circ y$ and $H$ is called intra-regular, if every element of $H$ is intra-regular.

Example 20. Let $H=\{x, y, z, w\}$ with the binary hyperoperation defined below:

\begin{tabular}{c|cccc}
$\circ$ & $x$ & $y$ & $z$ & $w$ \\
\hline$x$ & $x$ & $\{x, w\}$ & $\{x, w\}$ & $w$ \\
$y$ & $\{x, w\}$ & $\{y, z\}$ & $\{y, z\}$ & $w$ \\
$z$ & $\{x, w\}$ & $y$ & $y$ & $w$ \\
$w$ & $w$ & $w$ & $w$ & $w$
\end{tabular}

Clearly, $H$ is an LA-semihypergroup because the elements of $H$ satisfy the left invertive law. Here, $H$ is intra-regular because, $x \in\left(y \circ x^{2}\right) \circ z, y \in\left(z \circ y^{2}\right) \circ z, z \in\left(y \circ z^{2}\right) \circ y$, $w \in\left(x \circ w^{2}\right) \circ z$.

Example 21. Let $H=\mathbb{Z}$. Define a hyperoperation $\circ$ on $H$ by

$$
x \circ y=\{x, y\} \cup 2 \mathbb{Z} \quad \forall x, y \in H .
$$

Then, for all $x, y, z \in H$, we have

$$
(x \circ y) \circ z=\{x, y, z\} \cup 2 \mathbb{Z}=(z \circ y) \circ x
$$


This implies that $(H, \circ)$ is a an LA-semihypergroup. Since $x \in$ $(x \circ x) \circ x=\{x\} \cup 2 \mathbb{Z}$ and also $x \in(x \circ(x \circ x)) \circ x=\{x\} \cup 2 \mathbb{Z}$. Thus, $(H, \circ)$ is a regular as well as intra-regular LA-semihypergroup.

Definition 22. An element $a$ of an LA-semihypergroup $H$ with left identity $e$ is called a left (right) invertible if there exist $x \in H$ such that $e \in x \circ a(e \in a \circ x)$ and $a$ is called invertible if it is both a left and a right invertible. An LA-semihypergroup $H$ is called a left (right) invertible if every element of $H$ is a left (right) invertible and $H$ is called invertible if it is both a left and a right invertible.

Theorem 23. Every LA-semihypergroup $H$ with pure left identity e is intra-regular if $H$ is left (right) invertible.

Proof. Let $H$ be a left invertible LA-semihypergroup with pure left identity $e$. Then, for $a \in H$, there exist $a^{\prime} \in H$ such that $e \in a^{\prime} \circ a$. Now, by using left invertive law, and medial law, Lemmas 7 and 15, we have

$$
\begin{aligned}
a & =e \circ a=e \circ(e \circ a) \subseteq\left(a^{\prime} \circ a\right) \circ(e \circ a) \subseteq(H \circ a) \circ(H \circ a) \\
& =(H \circ a) \circ((H \circ H) \circ a)=(H \circ a) \circ((a \circ H) \circ H) \\
& =(a \circ H) \circ((H \circ a) \circ H)=(a \circ(H \circ a)) \circ(H \circ H) \\
& =(a \circ(H \circ a)) \circ H=(H \circ(a \circ a)) \circ H=\left(H \circ a^{2}\right) \circ H .
\end{aligned}
$$

This shows that $H$ is intra-regular. The case for right invertible can be seen in a similar way.

Theorem 24. An LA-semihypergroup $H$ with left identity e is intra-regular if $H \circ a=H$ or $a \circ H=H$ for all $a \in H$.

Proof. Let $H$ be an LA-semihypergroup such that $H \circ a=H$ holds for all $a \in H$. Then, $H=H^{2}$. Let $a \in H$, therefore, by using medial law, we have

$$
\begin{aligned}
a \in H & =(H \circ H) \circ H=((H \circ a) \circ(H \circ a)) \circ H \\
& =((H \circ H) \circ(a \circ a)) \circ H=\left(H \circ a^{2}\right) \circ H .
\end{aligned}
$$

This shows that $H$ is intra-regular.

Let $a \in H$ and assume that $a \circ H=H$ holds for all $a \in H$. Then, by using left invertive law, we have

$$
a \in H=H \circ H=(a \circ H) \circ H=(H \circ H) \circ a=H \circ a \text {. }
$$

Thus, $H \circ a=H$ holds for all $a \in H$; therefore, it follows from above that $H$ is intra-regular.

Corollary 25. An LA-semihypergroup $H$ with pure left identitye is intra-regular if $H \circ a=H$ or $a \circ H=H$ for all $a \in H$.

Corollary 26. If $H$ is an LA-semihypergroup such that $a \circ H=$ $H$ holds for all $a \in H$, then $H \circ a=H$ holds for all $a \in H$.

Theorem 27. If $H$ is an intra-regular $L A$-semihypergroup with pure left identity e, then $(B \circ H) \circ B=B$, where $B$ is a bi(generalized bi-) hyperideal of $H$.
Proof. Let $H$ be an intra-regular LA-semihypergroup with pure left identity $e$. Then, clearly, $(B \circ H) \circ B=B$. Now let $b \in B$. Since $H$ is intra-regular, so there exist $x, y \in H$ such that $b \in\left(x \circ b^{2}\right) \circ y$. Now, by using Lemma 7 , left invertive law, paramedial law and medial law, we have

$$
\begin{aligned}
b \in\left(x \circ b^{2}\right) \circ y & =(x \circ(b \circ b)) \circ y=(b \circ(x \circ b)) \circ y \\
& =(y \circ(x \circ b)) \circ b \\
& \subseteq\left(y \circ\left(x \circ\left(\left(x \circ b^{2}\right) \circ y\right)\right)\right) \circ b \\
& =\left(y \circ\left(\left(x \circ b^{2}\right) \circ(x \circ y)\right)\right) \circ b \\
& =\left(\left(x \circ b^{2}\right) \circ(y \circ(x \circ y))\right) \circ b \\
& =\left(((x \circ y) \circ y) \circ\left(b^{2} \circ x\right)\right) \circ b \\
& =((b \circ b) \circ(((x \circ y) \circ y) \circ x)) \circ b \\
& =((b \circ b) \circ((x \circ y) \circ(x \circ y))) \circ b \\
& =\left((b \circ b) \circ\left(x^{2} \circ y^{2}\right)\right) \circ b \\
& =\left(\left(y^{2} \circ x^{2}\right) \circ(b \circ b)\right) \circ b \\
& =\left(b \circ\left(\left(y^{2} \circ x^{2}\right) \circ b\right)\right) \circ b \subseteq(B \circ H) \circ B .
\end{aligned}
$$

This shows that $(B \circ H) \circ B=B$.

The converse is not true in general. For this, let us consider an LA-semihypergroup $H=\{x, y, z, w\}$ with the binary hyperoperation defined below:

\begin{tabular}{c|cccc}
$\circ$ & $x$ & $y$ & $z$ & $w$ \\
\hline$x$ & $y$ & $y$ & $\{z, w\}$ & $w$ \\
$y$ & $y$ & $y$ & $\{z, w\}$ & $w$ \\
$z$ & $\{z, w\}$ & $\{z, w\}$ & $z$ & $w$ \\
$w$ & $w$ & $w$ & $w$ & $w$
\end{tabular}

Clearly, $H$ is an LA-semihypergroup because the elements of $H$ satisfy the left invertive law. It is easy to see that $\{z, w\}$ is a bi-hyperideal of $H$ such that $(B \circ H) \circ B=B$, but $H$ has no pure left identity, and also $H$ is not an intra-regular because $x \in H$ is not an intra-regular.

Theorem 28. If $H$ is an intra-regular LA-semihypergroup with pure left identitye, then $(H \circ B) \circ H=B$, where $B$ is an interior hyperideal of $H$.

Proof. Let $H$ be an intra-regular LA-semihypergroup with pure left identity $e$. Then, clearly, $(H \circ B) \circ H=B$. Now, let $b \in B$. Since $H$ is an intra-regular, so there exist $x, y \in H$ such 
that $b \in\left(x \circ b^{2}\right) \circ y$. Now, by using paramedial law and left invertive law, we have

$$
\begin{aligned}
b \in\left(x \circ b^{2}\right) \circ y & =((e \circ x) \circ(b \circ b)) \circ y=((b \circ b) \circ(x \circ e)) \circ y \\
& =(((x \circ e) \circ b) \circ b) \circ y \subseteq(H \circ B) \circ H .
\end{aligned}
$$

This shows that $(H \circ B) \circ H=B$.

The converse is not true in general. For this, let us consider an LA-semihypergroup $H=\{e, x, y, z, w\}$ with the binary hyperoperation defined below:

\begin{tabular}{c|ccccc}
$\circ$ & $e$ & $x$ & $y$ & $z$ & $w$ \\
\hline$e$ & $e$ & $x$ & $y$ & $z$ & $w$ \\
$x$ & $y$ & $z$ & $z$ & $\{z, w\}$ & $w$ \\
$y$ & $x$ & $z$ & $z$ & $\{z, w\}$ & $w$ \\
$z$ & $z$ & $\{z, w\}$ & $\{z, w\}$ & $\{z, w\}$ & $w$ \\
$w$ & $w$ & $w$ & $w$ & $w$ & $w$
\end{tabular}

Clearly, $H$ is an LA-semihypergroup because the elements of $H$ satisfy the left invertive law. It is easy to see that $\{z, w\}$ is an interior hyperideal of $H$ with pure left identity $e$ such that $(H \circ\{z, w\}) \circ H=\{z, w\}$, but $H$ is not an intra-regular because $x \in H$, is not an intra-regular.

Definition 29. Let $H$ be an LA-semihypergroup. Then, $\emptyset \neq$ $A \subseteq H$ is called semiprime if $a^{2} \subseteq A$ implies $a \in A$.

Theorem 30. An LA-semihypergroup $H$ with pure left identity is intra-regular if $L \cup R=L \circ R$, where $L$ and $R$ are the left and right hyperideals of $H$, respectively, such that $R$ is semiprime.

Proof. Let $H$ be an LA-semihypergroup with pure left identity. Then, clearly, $H \circ a$ and $a^{2} \circ H$ are the left and right hyperideals of $H$ such that $a \in H \circ a$ and $a^{2} \subseteq a^{2} \circ H$, because by using paramedial law, we have

$$
a^{2} \circ H=(a \circ a) \circ(H \circ H)=(H \circ H) \circ(a \circ a)=H \circ a^{2} .
$$

Therefore, by the given assumption, $a \in a^{2} \circ H$. Now, by using left invertive law, medial law, paramedial law, and Lemma 7, we have

$$
\begin{aligned}
a \in H \circ a \cup a^{2} \circ H & =(H \circ a) \circ\left(a^{2} \circ H\right) \\
& =(H \circ a) \circ((a \circ a) \circ H) \\
& =(H \circ a) \circ((H \circ a) \circ(e \circ a)) \\
& \subseteq(H \circ a) \circ((H \circ a) \circ(H \circ a)) \\
& =(H \circ a) \circ((H \circ H) \circ(a \circ a)) \\
& \subseteq(H \circ a) \circ((H \circ H) \circ(H \circ a)) \\
& =(H \circ a) \circ((a \circ H) \circ(H \circ H)) \\
& =(H \circ a) \circ((a \circ H) \circ H) \\
& =(a \circ H) \circ((H \circ a) \circ H)
\end{aligned}
$$

$$
\begin{aligned}
& =(a \circ(H \circ a)) \circ(H \circ H) \\
& =(a \circ(H \circ a)) \circ H=(H \circ(a \circ a)) \circ H \\
& =\left(H \circ a^{2}\right) \circ H .
\end{aligned}
$$

This shows that $H$ is intra-regular.

Lemma 31. If $H$ is an intra-regular LA-semihypergroup, then $H=H^{2}$.

Proof. The proof is straightforward.

Theorem 32. For a left invertible LA-semihypergroup $H$ with pure left identity, the following conditions are equivalent:

(i) $H$ is intra-regular,

(ii) $R \cap L=R \circ L$, where $R$ and $L$ are any right and left hyperideals of $H$, respectively.

Proof. (i) $\Rightarrow$ (ii): assume that $H$ is an intra-regular LA-semihypergroup with pure left identity and let $a \in H$. Then, there exist $x, y \in H$ such that $a \in\left(x \circ a^{2}\right) \circ y$. Let $R$ and $L$ be any right and left hyperideals of $H$, respectively. Then, obviously, $R \circ L \subseteq R \cap L$. Now, let $a \in R \cap L$ imply that $a \in R$ and $a \in L$. Now by using medial law, left invertive law, and Lemma 7 , we have

$$
\begin{aligned}
a & \in\left(x \circ a^{2}\right) \circ y \subseteq\left(H \circ a^{2}\right) \circ H \\
& =(H \circ(a \circ a)) \circ H=(a \circ(H \circ a)) \circ H \\
& =(a \circ(H \circ a)) \circ(H \circ H) \\
& =(a \circ H) \circ((H \circ a) \circ H) \\
& =(H \circ a) \circ((a \circ H) \circ H) \\
& =(H \circ a) \circ((H \circ H) \circ a)=(H \circ a) \circ(H \circ a) \\
& \subseteq(H \circ R) \circ(H \circ L)=((H \circ H) \circ R) \circ(H \circ L) \\
& =((R \circ H) \circ H) \circ(H \circ L) \subseteq R \circ L .
\end{aligned}
$$

This shows that $R \cap L=R \circ L$.

(ii) $\Rightarrow$ (i): let $H$ be a left invertible LA-semihypergroup with pure left identity. Then, for $a \in H$. There, exists $a^{\prime} \in H$ such that $e \in a^{\prime} \circ a$. Since $a^{2} \circ H$ is a right hyperideal and also a left hyperideal of $H$ such that $a^{2} \subseteq a^{2} \circ H$, therefore, by using the given assumption, medial law, left invertive law, and Lemma 7, we have

$$
\begin{aligned}
a^{2} & \subseteq a^{2} \circ H \cap a^{2} \circ H=\left(a^{2} \circ H\right) \circ\left(a^{2} \circ H\right) \\
& =a^{2} \circ\left(\left(a^{2} \circ H\right) \circ H\right)=a^{2} \circ\left((H \circ H) \circ a^{2}\right) \\
& =(a \circ a) \circ\left(H \circ a^{2}\right)=\left(\left(H \circ a^{2}\right) \circ a\right) \circ a .
\end{aligned}
$$

Thus, we get $a^{2} \subseteq\left(\left(x \circ a^{2}\right) \circ a\right) \circ a$ for some $x \in H$. 
Now, by using left invertive law, we have

$$
\begin{aligned}
(a \circ a) \circ a^{\prime} \subseteq & \left(\left(\left(x \circ a^{2}\right) \circ a\right) \circ a\right) \circ a^{\prime} \\
\left(a^{\prime} \circ a\right) \circ a & \subseteq\left(a^{\prime} \circ a\right) \circ\left(\left(x \circ a^{2}\right) \circ a\right) \\
a & \in\left(x \circ a^{2}\right) \circ a .
\end{aligned}
$$

This shows that $H$ is intra-regular.

Lemma 33. Every left hyperideal of an intra-regular LA-semihypergroup $H$ with pure left identity is idempotent.

Proof. The proof is straightforward.

Theorem 34. In an LA-semihypergroup $H$ with pure left identity, the following conditions are equivalent:

(i) $H$ is intra-regular,

(ii) $A=(H \circ A)^{2}$, where $A$ is any left hyperideal of $H$.

Proof. (i) $\Rightarrow$ (ii): let $A$ be a left hyperideal of an intra-regular LA-semihypergroup $H$ with left identity. Then, $H \circ A \subseteq A$ and, by Lemma 33, $(H \circ A)^{2}=H \circ A \subseteq A$. Now $A=A \circ A \subseteq$ $H \circ A=(H \circ A)^{2}$, which implies that $A=(H \circ A)^{2}$.

(ii) $\Rightarrow$ (i): let $A$ be a left hyperideal of $H$. Then, $A=(H \circ$ $A)^{2} \subseteq A^{2}$, which implies that $A$ is idempotent; hence, $H$ is intra-regular.

Theorem 35. In an intra-regular LA-semihypergroup $H$ with pure left identity, the following conditions are equivalent:

(i) A is a bi- (generalized bi-) hyperideal of $H$,

(ii) $(A \circ H) \circ A=A$ and $A^{2}=A$.

Proof. (i) $\Rightarrow$ (ii): let $A$ be a bi-hyperideal of an intra-regular LA-semihypergroup $H$ with pure left identity. Then, $(A \circ H) \circ$ $A \subseteq A$. Let $a \in A$. Then, since $H$ is intra-regular, so there exist $x, y \in H$ such that $a \in\left(x \circ a^{2}\right) \circ y$. Now, by using medial, left invertive law, and Lemma 7, we have

$$
\begin{aligned}
a \in\left(x \circ a^{2}\right) \circ y & =(x \circ(a \circ a)) \circ y \\
& =(a \circ(x \circ a)) \circ y=(y \circ(x \circ a)) \circ a \\
& \subseteq\left(y \circ\left(x \circ\left(\left(x \circ a^{2}\right) \circ y\right)\right)\right) \circ a \\
& =\left(y \circ\left(\left(x \circ a^{2}\right) \circ(x \circ y)\right)\right) \circ a \\
& =\left(\left(x \circ a^{2}\right) \circ(y \circ(x \circ y))\right) \circ a \\
& =((x \circ(a \circ a)) \circ(y \circ(x \circ y))) \circ a \\
& =((a \circ(x \circ a)) \circ(y \circ(x \circ y))) \circ a \\
& =((a \circ y) \circ((x \circ a) \circ(x \circ y))) \circ a
\end{aligned}
$$

$$
\begin{aligned}
& =((x \circ a) \circ((a \circ y) \circ(x \circ y))) \circ a \\
& =\left((x \circ a) \circ\left((a \circ x) \circ y^{2}\right)\right) \circ a \\
& =\left(\left(y^{2} \circ(a \circ x)\right) \circ(a \circ x)\right) \circ a \\
& =\left(a \circ\left(\left(y^{2} \circ(a \circ x)\right) \circ x\right)\right) \circ a \\
& \subseteq(A \circ H) \circ A .
\end{aligned}
$$

Thus, $(A \circ H) \circ A=A$ holds. Now, by using left invertive law, paramedial law, medial law, and Lemma 7 , we have

$$
\begin{aligned}
& a \in\left(x \circ a^{2} \circ\right) \circ y=(x \circ(a \circ a)) \circ y \\
& =(a \circ(x \circ a)) \circ y=(y \circ(x \circ a)) \circ a \\
& \subseteq\left(y \circ\left(x \circ\left(\left(x \circ a^{2}\right) \circ y\right)\right)\right) \circ a \\
& =\left(y \circ\left(\left(x \circ a^{2}\right) \circ(x \circ y)\right)\right) \circ a \\
& =\left(\left(x \circ a^{2}\right) \circ(y \circ(x \circ y))\right) \circ a \\
& =((x \circ(a \circ a)) \circ(y \circ(x \circ y))) \circ a \\
& =((a \circ(x \circ a)) \circ(y \circ(x \circ y))) \circ a \\
& =(((y \circ(x \circ y)) \circ(x \circ a)) \circ a) \circ a \\
& =(((a \circ x) \circ((x \circ y) \circ y)) \circ a) \circ a \\
& =\left(\left((a \circ x) \circ\left(y^{2} \circ x\right)\right) \circ a\right) \circ a \\
& =\left(\left(\left(a \circ y^{2}\right) \circ(x \circ x)\right) \circ a\right) \circ a \\
& =\left(\left(\left(a \circ y^{2}\right) \circ x^{2}\right) \circ a\right) \circ a \\
& =\left(\left(\left(x^{2} \circ y^{2}\right) \circ a\right) \circ a\right) \circ a \\
& \subseteq\left(\left(\left(x^{2} \circ y^{2}\right) \circ((x \circ(a \circ a)) \circ y)\right) \circ a\right) \circ a \\
& =\left(\left(\left(x^{2} \circ y^{2}\right) \circ((a \circ(x \circ a)) \circ y)\right) \circ a\right) \circ a \\
& =\left(\left(\left(x^{2} \circ(a \circ(x \circ a))\right) \circ\left(y^{2} \circ y\right)\right) \circ a\right) \circ a \\
& =\left(\left(\left(a \circ\left(x^{2} \circ(x \circ a)\right)\right) \circ y^{3}\right) \circ a\right) \circ a \\
& =\left(\left((a \circ((x \circ x) \circ(x \circ a))) \circ y^{3}\right) \circ a\right) \circ a \\
& =\left(\left((a \circ((a \circ x) \circ(x \circ x))) \circ y^{3}\right) \circ a\right) \circ a \\
& =\left(\left(\left((a \circ x) \circ\left(a \circ x^{2}\right)\right) \circ y^{3}\right) \circ a\right) \circ a \\
& =\left(\left(\left((a \circ a) \circ\left(x \circ x^{2}\right)\right) \circ y^{3}\right) \circ a\right) \circ a \\
& =\left(\left(\left(y^{3} \circ x^{3}\right) \circ(a \circ a)\right) \circ a\right) \circ a
\end{aligned}
$$




$$
\begin{aligned}
& =\left(\left(a \circ\left(\left(y^{3} \circ x^{3}\right) \circ a\right)\right) \circ a\right) \circ a \\
& \subseteq((A \circ H) \circ A) \circ A \subseteq A \circ A=A^{2} .
\end{aligned}
$$

Hence, $A=A^{2}$ holds.

(ii) $\Rightarrow$ (i) is obvious.

Theorem 36. In an intra-regular LA-semihypergroup $H$ with pure left identity, the following conditions are equivalent:

(i) $A$ is a quasi-hyperideal of $H$,

(ii) $H \circ Q \cap Q \circ H=Q$.

Proof. (i) $\Rightarrow$ (ii): let $Q$ be a quasi hyperideal of an intraregular LA-semihypergroup $H$ with pure left identity. Then, $H \circ Q \cap Q \circ H \subseteq Q$. Let $q \in Q$, then since $H$ is intra-regular, so there exist $x, y \in H$ such that $q \in\left(x \circ q^{2}\right) \circ y$. Let $p \circ q \subseteq H \circ Q$. Then, by using medial law, paramedial law and Lemma 7 , we have

$$
\begin{aligned}
p \circ q & \subseteq p \circ\left(\left(x \circ q^{2}\right) \circ y\right)=\left(x \circ q^{2}\right) \circ(p \circ y) \\
& =(x \circ(q \circ q)) \circ(p \circ y)=(q \circ(x \circ q)) \circ(p \circ y) \\
& =(q \circ p) \circ((x \circ q) \circ y)=(x \circ q) \circ((q \circ p) \circ y) \\
& =(y \circ(q \circ p)) \circ(q \circ x)=q \circ((y \circ(q \circ p)) \circ x) \\
& \subseteq Q \circ H .
\end{aligned}
$$

Now, let $q \circ p \in Q \circ H$. Then, by using medial law, paramedial law and Lemma 7 , we have

$$
\begin{aligned}
q \circ p & \subseteq\left(\left(x \circ q^{2}\right) \circ y\right) \circ p=(p \circ y) \circ\left(x \circ q^{2}\right) \\
& =(p \circ y) \circ(x \circ(q \circ q))=x \circ((p \circ y) \circ(q \circ q)) \\
& =x \circ((q \circ q) \circ(y \circ p))=(q \circ q) \circ(x \circ(y \circ p)) \\
& =((x \circ(y \circ p)) \circ q) \circ q \subseteq H \circ Q .
\end{aligned}
$$

Hence, $Q \circ H=H \circ Q$. As, by using left invertive law, medial law, and Lemma 7, we have

$$
\begin{aligned}
q \in\left(x \circ q^{2}\right) \circ y & =(x \circ(q \circ q)) \circ y=(q \circ(x \circ q)) \circ y \\
& =(y \circ(x \circ q)) \circ q \subseteq H \circ Q .
\end{aligned}
$$

Thus, $q \in H \circ Q \cap Q \circ H$ implies that $H \circ Q \cap Q \circ H=Q$.

(ii) $\Rightarrow$ (i) is obvious.

Theorem 37. In an intra-regular LA-semihypergroup $H$ with pure left identity, the following conditions are equivalent:

(i) $A$ is an interior hyperideal of $H$,

(ii) $(H \circ A) \circ H=A$.

Proof. (i) $\Rightarrow$ (ii): let $A$ be an interior hyperideal of an intraregular LA-semihypergroup $H$ with pure left identity. Then,
$(H \circ A) \circ H \subseteq A$. Let $a \in A$, then since $H$ is intra-regular, so there exist $x, y \in H$ such that $a \in\left(x \circ a^{2}\right) \circ y$. Now, by using left invertive law, medial law, paramedial law, and Lemma 7, we have

$$
\begin{aligned}
a \in\left(x \circ a^{2}\right) \circ y & =(x \circ(a \circ a)) \circ y \\
& =(a \circ(x \circ a)) \circ y=(y \circ(x \circ a)) \circ a \\
& \subseteq(y \circ(x \circ a)) \circ\left(\left(x \circ a^{2}\right) \circ y\right) \\
& =\left(\left(\left(x \circ a^{2}\right) \circ y\right) \circ(x \circ a)\right) \circ y \\
& =\left((a \circ x) \circ\left(y \circ\left(x \circ a^{2}\right)\right)\right) \circ y \\
& =\left(\left(\left(y \circ\left(x \circ a^{2}\right)\right) \circ x\right) \circ a\right) \circ y \\
& \subseteq(H \circ A) \circ H .
\end{aligned}
$$

Thus, $(H \circ A) \circ H=A$.

(ii) $\Rightarrow$ (i) is obvious.

Theorem 38. In an intra-regular LA-semihypergroup $H$ with pure left identity, the following conditions are equivalent.

(i) $A$ is a $(1,2)$-hyperideal of $H$,

(ii) $(A \circ H) \circ A^{2}=A$ and $A^{2}=A$.

Proof. (i) $\Rightarrow$ (ii): let $A$ be a $(1,2)$-hyperideal of an intraregular LA-semihypergroup $H$ with pure left identity. Then, $(A \circ H) \circ A^{2} \subseteq A$ and $A^{2} \subseteq A$. Let $a \in A$. Then, since $H$ is intra-regular, so there exist $x, y \in H$ such that $a \in\left(x \circ a^{2}\right) \circ y$. Now, by using left invertive law, medial law, paramedial law and Lemma 7, we have

$$
\begin{aligned}
a \in\left(x \circ a^{2}\right) \circ y & =(x \circ(a \circ a)) \circ y \\
& =(a \circ(x \circ a)) \circ y=(y \circ(x \circ a)) \circ a \\
& \subseteq\left(y \circ\left(x \circ\left(\left(x \circ a^{2}\right) \circ y\right)\right)\right) \circ a \\
& =\left(y \circ\left(\left(x \circ a^{2}\right) \circ(x \circ y)\right)\right) \circ a \\
& =\left(\left(x \circ a^{2}\right) \circ(y \circ(x \circ y))\right) \circ a \\
& =\left(((x \circ y) \circ y) \circ\left(a^{2} \circ x\right)\right) \circ a \\
& =\left(\left(y^{2} \circ x\right) \circ\left(a^{2} \circ x\right)\right) \circ a \\
& =\left(a^{2} \circ\left(\left(y^{2} \circ x\right) \circ x\right)\right) \circ a \\
& =\left(a^{2} \circ\left(x^{2} \circ y^{2}\right)\right) \circ a \\
& =\left(a \circ\left(x^{2} \circ y^{2}\right)\right) \circ a^{2} \\
& =\left(a \circ\left(x^{2} \circ y^{2}\right)\right) \circ(a \circ a) \subseteq(A \circ H) \circ A^{2} .
\end{aligned}
$$


Thus, $(A \circ H) \circ A^{2}=A$. Now, by using left invertive law, medial law, paramedial law, and Lemma 7, we have

$$
\begin{aligned}
& a \in\left(x \circ a^{2}\right) \circ y=(x \circ(a \circ a)) \circ y \\
& =(a \circ(x \circ a)) \circ y=(y \circ(x \circ a)) a \\
& \subseteq(y \circ(x \circ a)) \circ\left(\left(x \circ a^{2}\right) \circ y\right) \\
& =\left(x \circ a^{2}\right) \circ((y \circ(x \circ a)) \circ y) \\
& =(x \circ(a \circ a)) \circ((y \circ(x \circ a)) \circ y) \\
& =(a \circ(x \circ a)) \circ((y \circ(x \circ a)) \circ y) \\
& =(((y \circ(x \circ a)) \circ y) \circ(x \circ a)) \circ a \\
& =((a \circ x) \circ(y \circ(y \circ(x \circ a)))) \circ a \\
& =\left(\left(\left(\left(x \circ a^{2}\right) \circ y\right) \circ x\right) \circ(y \circ(y \circ(x \circ a)))\right) \circ a \\
& =\left(\left((x \circ y) \circ\left(x \circ a^{2}\right)\right) \circ(y \circ(y \circ(x \circ a)))\right) \circ a \\
& =\left(((x \circ y) \circ y) \circ\left(\left(x \circ a^{2}\right) \circ(y \circ(x \circ a))\right)\right) \circ a \\
& =\left(\left(y^{2} \circ x\right) \circ((x \circ(a \circ a)) \circ(y \circ(x \circ a)))\right) \circ a \\
& =\left(\left(y^{2} \circ x\right) \circ((x \circ y) \circ((a \circ a) \circ(x \circ a)))\right) \circ a \\
& =\left(\left(y^{2} \circ x\right) \circ((a \circ a) \circ((x \circ y) \circ(x \circ a)))\right) \circ a \\
& =\left((a \circ a) \circ\left(\left(y^{2} \circ x\right) \circ((x \circ y) \circ(x \circ a))\right)\right) \circ a \\
& =\left((a \circ a) \circ\left(\left(y^{2} \circ x\right) \circ((x \circ x) \circ(y \circ a))\right)\right) \circ a \\
& =\left(\left(((x \circ x) \circ(y \circ a)) \circ\left(y^{2} \circ x\right)\right) \circ(a \circ a)\right) \circ a \\
& =\left(\left(((a \circ y) \circ(x \circ x)) \circ\left(y^{2} \circ x\right)\right) \circ(a \circ a)\right) \circ a \\
& =\left(\left(\left(\left(x^{2} \circ y\right) \circ a\right) \circ\left(y^{2} \circ x\right)\right) \circ(a \circ a)\right) \circ a \\
& =\left(\left(\left(x \circ y^{2}\right) \circ\left(a \circ\left(x^{2} \circ y\right)\right)\right) \circ(a \circ a)\right) \circ a \\
& =\left(\left(a \circ\left(\left(x \circ y^{2}\right) \circ\left(x^{2} \circ y\right)\right)\right) \circ(a \circ a)\right) \circ a \\
& =\left(\left(a \circ\left(x^{3} \circ y^{3}\right)\right) \circ(a \circ a)\right) \circ a \\
& \subseteq\left((A \circ H) \circ A^{2}\right) \circ A \subseteq A \circ A=A^{2} .
\end{aligned}
$$

Hence, $A^{2}=A$.

(ii) $\Rightarrow$ (i) is obvious.

Lemma 39. Every nonempty subset A of an intra-regular LAsemihypergroup $H$ with pure left identity is a left hyperideal of $H$ if and only if it is a right hyperideal of $H$.

Proof. The proof is straightforward.
Theorem 40. In an intra-regular LA-semihypergroup $H$ with pure left identity, the following conditions are equivalent:

(i) $A$ is a $(1,2)$-hyperideal of $H$,

(ii) $A$ is a two-sided hyperideal of $H$.

Proof. (i) $\Rightarrow$ (ii): assume that $H$ is intra-regular LAsemihypergroup with pure left identity, and let $A$ be a $(1,2)$ hyperideal of $H$. Then, $(A \circ H) \circ A^{2} \subseteq A$. Let $a \in A$. Then, since $H$ is intra-regular, so there exist $x, y \in H$ such that $a \in\left(x \circ a^{2}\right) \circ y$. Now, by using left invertive law, medial law, paramedial law, and Lemma 7 , we have

$$
\begin{aligned}
& H \circ a \subseteq H \circ\left(\left(x \circ a^{2}\right) \circ y\right)=\left(x \circ a^{2}\right) \circ(H \circ y) \\
& =(x \circ(a \circ a)) \circ(H \circ y)=(a \circ(x \circ a)) \circ(H \circ y) \\
& =((H \circ y) \circ(x \circ a)) \circ a \\
& \subseteq((H \circ y) \circ(x \circ a)) \circ\left(\left(x \circ a^{2}\right) \circ y\right) \\
& =\left(x \circ a^{2}\right) \circ(((H \circ y) \circ(x \circ a)) \circ y) \\
& =(y \circ((H \circ y) \circ(x \circ a))) \circ\left(a^{2} \circ x\right) \\
& =a^{2} \circ((y \circ((H \circ y) \circ(x \circ a))) \circ x) \\
& =(a \circ a) \circ((y \circ((H \circ y) \circ(x \circ a))) \circ x) \\
& =(x \circ(y \circ((H \circ y) \circ(x \circ a)))) \circ(a \circ a) \\
& =(x \circ(y \circ((a \circ x) \circ(y \circ H)))) \circ(a \circ a) \\
& =(x \circ((a \circ x) \circ(y \circ(y \circ H)))) \circ(a \circ a) \\
& =((a \circ x) \circ(x \circ(y \circ(y \circ H)))) \circ(a \circ a) \\
& \subseteq\left(\left(\left(\left(x \circ a^{2}\right) \circ y\right) \circ x\right) \circ(x \circ(y \circ(y \circ H)))\right) \circ(a \circ a) \\
& =\left(\left((x \circ y) \circ\left(x \circ a^{2}\right)\right) \circ(x \circ(y \circ(y \circ H)))\right) \circ(a \circ a) \\
& =\left(\left(\left(a^{2} \circ x\right) \circ(y \circ x)\right) \circ(x \circ(y \circ(y \circ H)))\right) \circ(a \circ a) \\
& =\left(\left(((y \circ x) \circ x) \circ a^{2}\right) \circ(x \circ(y \circ(y \circ H)))\right) \circ(a \circ a) \\
& =\left(((y \circ(y \circ H)) \circ x) \circ\left(a^{2} \circ((y \circ x) \circ x)\right)\right) \circ(a \circ a) \\
& =\left(((y \circ(y \circ H)) \circ x) \circ\left(a^{2} \circ\left(x^{2} \circ y\right)\right)\right) \circ(a \circ a) \\
& =\left(a^{2} \circ\left(((y \circ(y \circ H)) \circ x) \circ\left(x^{2} \circ y\right)\right)\right) \circ(a \circ a) \\
& =\left((a \circ a) \circ\left(((y \circ(y \circ H)) \circ x) \circ\left(x^{2} \circ y\right)\right)\right) \circ(a \circ a) \\
& =\left(\left(\left(x^{2} \circ y\right) \circ((y \circ(y \circ H)) \circ x)\right) \circ(a \circ a)\right) \circ(a \circ a) \\
& =\left(a \circ\left(\left(x^{2} \circ y\right) \circ(((y \circ(y \circ H)) \circ x) \circ a)\right)\right) \circ(a \circ a) \\
& \subseteq(A \circ H) \circ A^{2} \subseteq A \text {. }
\end{aligned}
$$


Hence, $A$ is a left hyperideal of $H$, and, by Lemma 39, $A$ is a two-sided hyperideal of $H$.

(ii) $\Rightarrow$ (i): let $A$ be a two-sided hyperideal of $H$. Let $y \in$ $(A \circ H) \circ A^{2}$. Then, $y \in(a \circ H) \circ b^{2}$ for some $a, b \in A$. Now, by using Lemma 7 , we have

$$
\begin{aligned}
y \in(a \circ H) \circ b^{2} & =(a \circ H) \circ(b \circ b) \\
& =b \circ((a \circ H) \circ b) \subseteq A \circ H \subseteq A .
\end{aligned}
$$

Hence, $(A \circ H) \circ A^{2} \subseteq A$; therefore, $A$ is a $(1,2)$-hyperideal of $H$.

Lemma 41. Every nonempty subset $A$ of an intra-regular LA-semihypergroup $H$ with pure left identity is a two-sided hyperideal of $H$ if and only if it is a quasi hyperideal of $H$.

Proof. The proof is straightforward.

Theorem 42. A two-sided hyperideal of an intra-regular LAsemihypergroup $H$ with pure left identity is minimal if and only if it is the intersection of two minimal two-sided hyperideals of $H$.

Proof. Let $H$ be an intra-regular LA-semihypergroup and $Q$ a minimal two-sided hyperideal of $H$. Let $a \in Q$. As $H \circ(H \circ a) \subseteq$ $H \circ a$ and $H \circ(a \circ H) \subseteq a \circ(H \circ H)=a \circ H$, which shows that $H \circ a$ and $a \circ H$ are left hyperideals of $H$. So, by Lemma 39, $H \circ a$ and $a \circ H$ are two-sided hyperideals of $H$. Now,

$$
\begin{aligned}
H \circ & (H \circ a \cap a \circ H) \cap(H \circ a \cap a \circ H) \circ H \\
& =H \circ(H \circ a) \cap H \circ(a \circ H) \\
& \cap(H \circ a) \circ H \cap(a \circ H) \circ H \\
& \subseteq(H \circ a \cap a \circ H) \cap(H \circ a) \circ H \\
& \cap H \circ a \subseteq H \circ a \cap a \circ H .
\end{aligned}
$$

This implies that $H \circ a \cap a \circ H$ is a quasi hyperideal of $H$, so by using Lemma $41, H \circ a \cap a \circ H$ is a two-sided hyperideal of $H$. Also since $a \in Q$, we have

$$
H \circ a \cap a \circ H \subseteq H \circ Q \cap Q \circ H \subseteq Q \cap Q \subseteq Q
$$

Now, since $Q$ is minimal, so $H \circ a \cap a \circ H=Q$, where $H \circ a$ and $a \circ H$ are minimal two-sided hyperideals of $H$. Let, $I$ be any two-sided hyperideal of $H$ such that $I \subseteq H \circ a$. Then, $I \cap a \circ H \subseteq H \circ a \cap a \circ H \subseteq Q$, which implies that $I \cap a \circ H=Q$. Thus $Q \subseteq I$. Therefore, we have

$$
H \circ a \subseteq H \circ Q \subseteq H \circ I \subseteq I \text {, and gives } H \circ a=I \text {. }
$$

Thus, $H \circ a$ is a minimal two-sided hyperideal of $H$. Similarly, $a \circ H$ is a minimal two-sided hyperideal of $H$.

Conversely, let $Q=I \cap J$ be a two-sided hyperideal of $H$, where $I$ and $J$ are minimal two-sided hyperideals of $H$. Then, by using Lemma $41, Q$ is a quasi hyperideal of $H$; that is, $H \circ Q \cap Q \circ H \subseteq Q$. Let $Q^{\prime}$ be a two-sided hyperideal of $H$ such that $Q^{\prime} \subseteq Q$. Then,

$$
\begin{aligned}
& H \circ Q^{\prime} \cap Q^{\prime} \circ H \subseteq H \circ Q \cap Q \circ H \subseteq Q, \\
& \text { also } H \circ Q^{\prime} \subseteq H \circ I \subseteq I, \\
& Q^{\prime} \circ H \subseteq J \circ H \subseteq J .
\end{aligned}
$$

Now

$$
\begin{aligned}
H \circ\left(H \circ Q^{\prime}\right) & =(H \circ H) \circ\left(H \circ Q^{\prime}\right) \\
& =\left(Q^{\prime} \circ H\right) \circ(H \circ H) \\
& =\left(Q^{\prime} \circ H\right) \circ H \\
& =(H \circ H) \circ Q^{\prime}=H \circ Q^{\prime},
\end{aligned}
$$

which implies that $H \circ Q^{\prime}$ is a left hyperideal and, hence, a twosided hyperideal by Lemma 39. Similarly, $Q^{\prime} \circ H$ is a two-sided hyperideal of $H$.

But since $I$ and $J$ are minimal two-sided hyperideals of $H$, therefore, $H \circ Q^{\prime}=I$ and $Q^{\prime} \circ H=J$. But $Q=I \cap J$, which implies that $Q=H \circ Q^{\prime} \cap Q^{\prime} \circ H \subseteq Q^{\prime}$. This gives us that $Q=Q^{\prime}$ and, hence, $Q$ is minimal.

\section{References}

[1] M. A. Kazim and M. Naseeruddin, "On almost semigroups," The Aligarh Bulletin of Mathematics, vol. 2, pp. 1-7, 1972.

[2] Q. Mushtaq and S. M. Yusuf, "On LA-semigroups," The Aligarh Bulletin of Mathematics, vol. 8, pp. 65-70, 1978.

[3] Q. Mushtaq and M. Khan, "M-systems in LA-semigroups," Southeast Asian Bulletin of Mathematics, vol. 33, no. 2, pp. 321327, 2009.

[4] P. Holgate, "Groupoids satisfying a simple invertive law," The Mathematics Student, vol. 61, no. 1-4, pp. 101-106, 1992.

[5] J. R. Cho, J. Ježek, and T. Kepka, "Paramedial groupoids," Czechoslovak Mathematical Journal, vol. 49, no. 2, pp. 277-290, 1999.

[6] M. Akram, N. Yaqoob, and M. Khan, "On $(m, n)$-ideals in LAsemigroups," Applied Mathematical Sciences, vol. 7, no. 44, pp. 2187-2191, 2013.

[7] M. Khan and N. Ahmad, "Characterizations of left almost semigroups by their ideals," Journal of Advanced Research in Pure Mathematics, vol. 2, no. 3, pp. 61-73, 2010.

[8] P. V. Protić and N. Stevanović, "AG-test and some general properties of Abel-Grassmann's groupoids," Pure Mathematics and Applications, vol. 6, no. 4, pp. 371-383, 1995.

[9] N. Stevanović and P. V. Protić, "Composition of Abel-Grassmann's 3-bands," Novi Sad Journal of Mathematics, vol. 34, no. 2, pp. 175-182, 2004.

[10] Q. Mushtaq and S. M. Yusuf, "On locally associative LAsemigroups," The Journal of Natural Sciences and Mathematics, vol. 19, no. 1, pp. 57-62, 1979.

[11] F. Marty, "Sur une generalization de la notion de groupe, $8^{\text {iem }}$," Congrès des Mathématiciens Scandinaves Tenu à Stockholm, pp. 45-49, 1934.

[12] P. Corsini, Prolegomena of Hypergroup Theory, Aviani Editore, 1993. 
[13] T. Vougiouklis, Hyperstructures and Their Representations, Hadronic Press, Palm Harbor, Fla, USA, 1994.

[14] P. Corsini and V. Leoreanu, Applications of Hyperstructure Theory, Kluwer Academic, 2003.

[15] B. Davvaz and V. L. Fotea, Hyperring Theory and Applications, International Academic Press, Gulf Breeze, Fla, USA, 2007.

[16] M. Aslam, T. Aroob, and N. Yaqoob, "On cubic $\Gamma$-hyperideals in left almost $\Gamma$-semihypergroups," Annals of Fuzzy Mathematics and Informatics, vol. 5, no. 1, pp. 169-182, 2013.

[17] M. Aslam, S. Abdullah, B. Davvaz, and N. Yaqoob, "Rough M-hypersystems and fuzzy Mhypersystems in $\Gamma$-semihypergroups," Neural Computing and Applicstions, vol. 21, no. 1, pp. 281-287, 2012.

[18] A. Ahmed, M. Aslam, and S. Abdullah, " $(\alpha, \beta)$-fuzzy hyperideals of semihyperring," World Applied Sciences Journal, vol. 18, no. 11, pp. 1501-1511, 2012.

[19] S. Abdullah, M. Aslam, and T. Anwar, "A note on M-hypersystems and N-hypersystems in $\Gamma$-semihypergroups," Quasigroups and Related Systems, vol. 19, no. 2, pp. 169-172, 2011.

[20] S. Abdullah, K. Hila, and M. Aslam, "On bi- $\Gamma$-hyperideals of $\Gamma$ semihypergroups," U.P.B. Scientific Bulletin, Series A, vol. 74, no. 4, pp. 79-90, 2012.

[21] P. Bonansinga and P. Corsini, "On semihypergroup and hypergroup homomorphisms," Bollettino dell'Unione Matematica Italiana A, vol. 1, no. 2, pp. 717-727, 1982.

[22] P. Corsini and I. Cristea, "Fuzzy sets and non complete 1hypergroups," Analele Stiintifice ale Universitatii Ovidius Constanta, vol. 13, no. 1, pp. 27-53, 2005.

[23] B. Davvaz, "Some results on congruences on semihypergroups," Bulletin of the Malaysian Mathematical Sciences Society, vol. 23, no. 1, pp. 53-58, 2000.

[24] B. Davvaz and N. S. Poursalavati, "Semihypergroups and Shypersystems," Pure Mathematics and Applications, vol. 11, no. 1, pp. 43-49, 2000.

[25] A. Hasankhani, "Ideals in a semihypergroup and Green's relations," Ratio Mathematica. Journal of Applied Mathematics, vol. 13, pp. 29-36, 1999.

[26] K. Hila, B. Davvaz, and K. Naka, "On quasi-hyperideals in semihypergroups," Communications in Algebra, vol. 39, no. 11, pp. 4183-4194, 2011.

[27] V. Leoreanu, "About the simplifiable cyclic semihypergroups," Italian Journal of Pure and Applied Mathematics, no. 7, pp. 69$76,2000$.

[28] S. V. Onipchuk, "Regular semihypergroups," Matematicheskiu Sbornik, vol. 183, no. 6, pp. 43-54, 1992.

[29] N. Yaqoob, M. Aslam, B. Davvaz, and A. B. Saeid, "On rough $(m, n)$ bi- $\Gamma$-hyperideals in $\Gamma$-semihypergroups," U.P.B. Scientific Bulletin, Series A, vol. 75, no. 1, pp. 119-128, 2013.

[30] N. Yaqoob, "Applications of rough sets to $\Gamma$-hyperideals in left almost $\Gamma$-semihypergroups," Neural Computing and Applications, vol. 21, no. 1, pp. 267-273, 2012.

[31] N. Yaqoob, M. Aslam, and M. A. Ansari, "Structures of N-Гhyperideals in left almost $\Gamma$-semihypergroups," World Applied Sciences Journal, vol. 17, no. 12, pp. 1611-1617, 2012.

[32] N. Yaqoob, M. Aslam, and K. Hila, "Rough fuzzy hyperideals in ternary semihypergroups," Advances in Fuzzy Systems, vol. 2012, Article ID 595687, 9 pages, 2012.

[33] N. Yaqoob and M. Aslam, "On bi- $\Gamma$-hyperideals in left almost $\Gamma$-semihypergroups," Journal of Advanced Research in Pure Mathematics, vol. 4, no. 1, pp. 130-143, 2012.
[34] N. Yaqoob, S. Abdullah, Faisal, and S. M. Qurashi, "On bi- hyperideals and M-hypersystems in left almost $\Gamma$-semihypergroups," International Journal of Algebra and Statistics, vol. 1, no. 1, pp. 1-7, 2012.

[35] K. Hila and J. Dine, "On hyperideals in left almost semihypergroups,” ISRN Algebra, vol. 2011, Article ID 953124, 8 pages, 2011. 


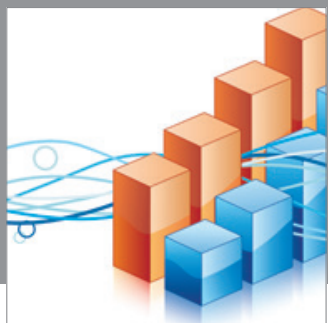

Advances in

Operations Research

mansans

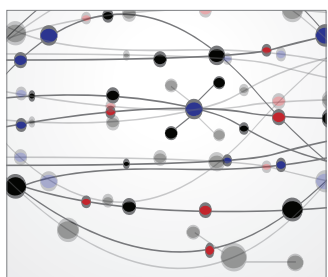

The Scientific World Journal
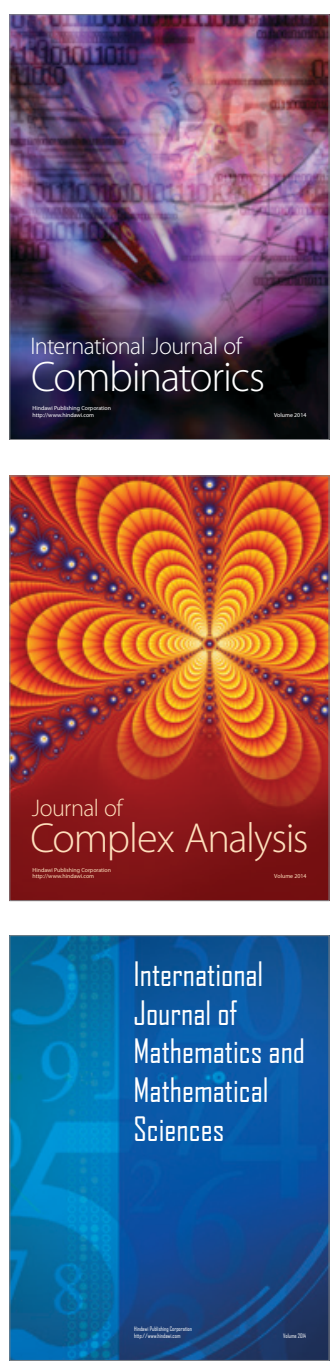
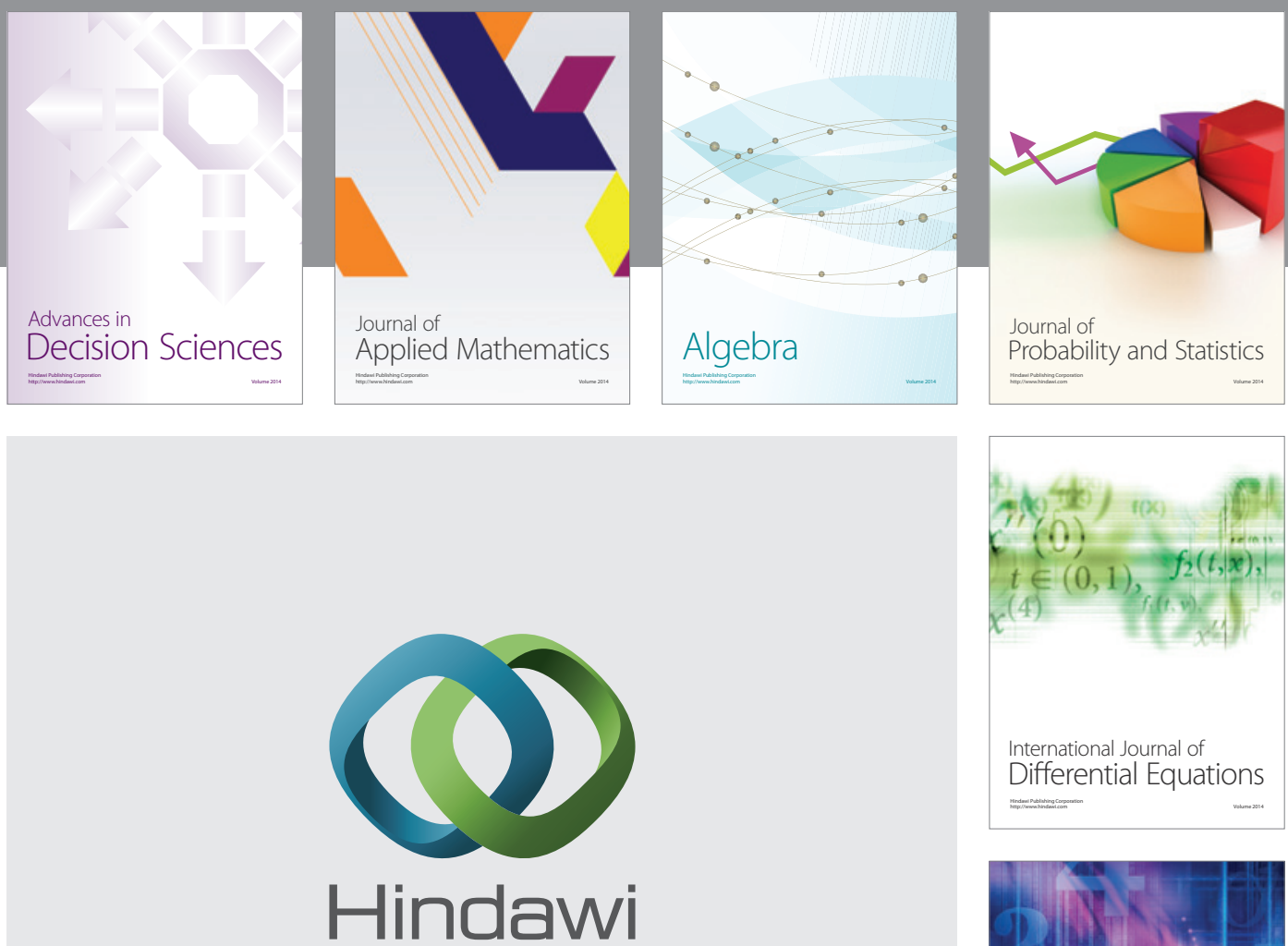

Submit your manuscripts at http://www.hindawi.com
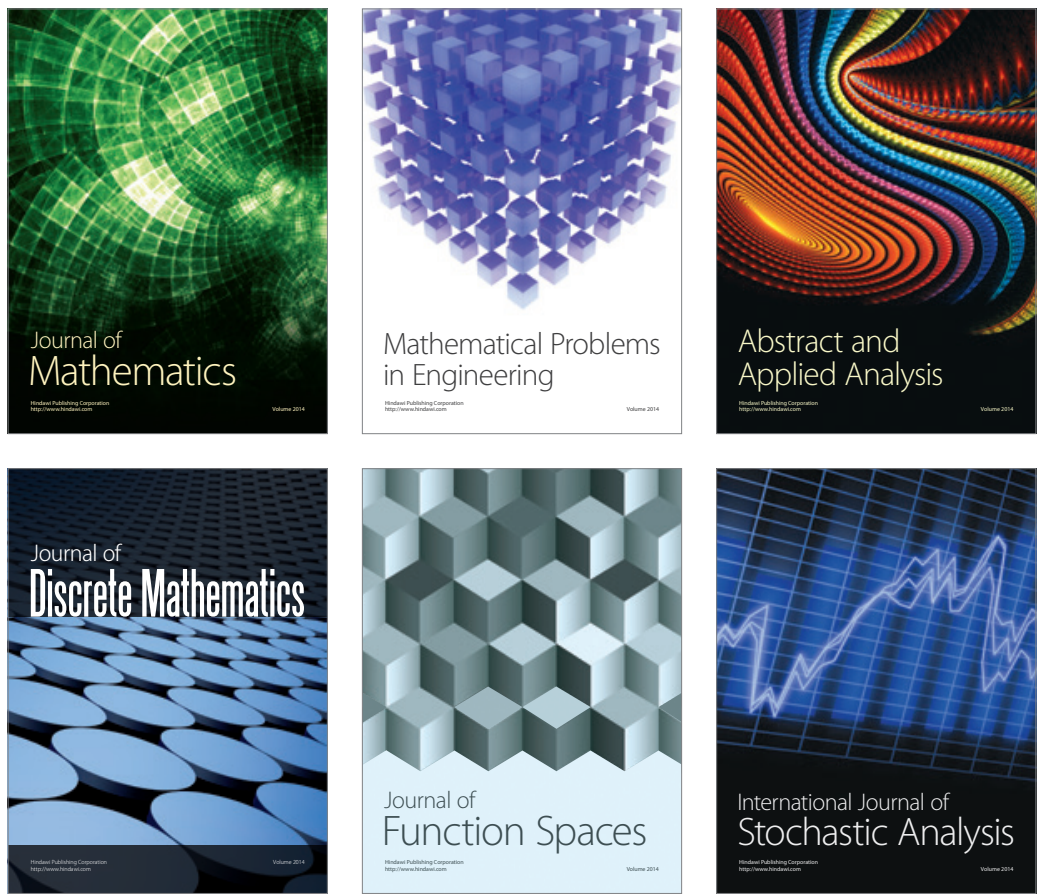

Journal of

Function Spaces

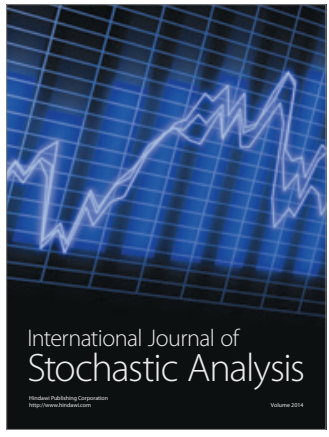

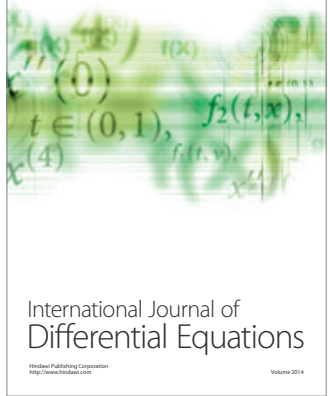
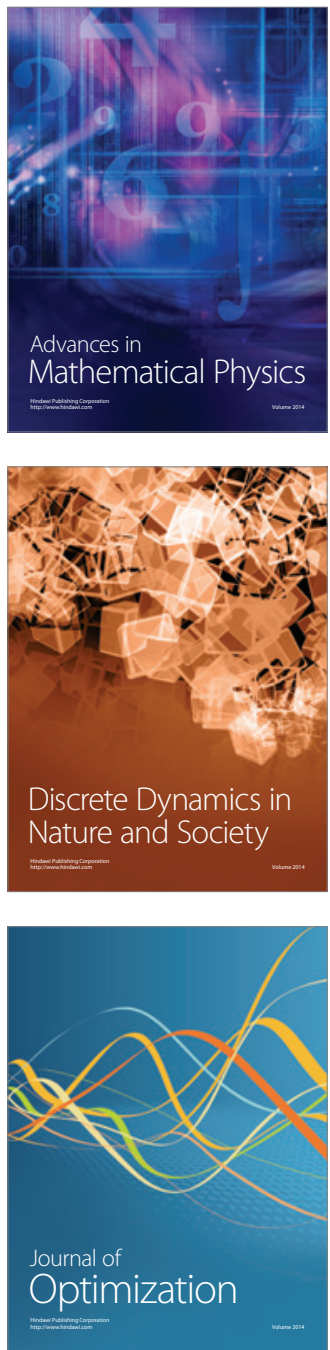